\title{
Transvaginal placement of surgical mesh for pelvic organ prolapse: more FDA concerns-positive reactions are possible
}

\author{
Bernard T. Haylen • Peter K. Sand • Steven E. Swift • \\ Christopher Maher • Paul A. Moran • \\ Robert M. Freeman
}

Received: 8 August 2011 / Accepted: 30 September 2011 /Published online: 16 November 2011

(C) The International Urogynecological Association 2011

Recently (July 13, 2011), the United States Food and Drug Administration (FDA) issued a document entitled "FDA Safety Communication: UPDATE on Serious Complications Associated with Transvaginal Placement of Surgical Mesh for Pelvic Organ Prolapse" [1]. This was stated as an update of a previous document issued on October 20, 2008,

Further contributions to this debate can be found at doi:10.1007/ s00192-011-1581-2, doi:10.1007/s00192-011-1596-8 and entitled "A Public Health Notification and Additional Patient Information on serious complications associated with surgical mesh placed through the vagina (transvaginal placement) to treat pelvic organ prolapse (POP) and SUI (stress urinary incontinence)". Accompanying the recent FDA concerns was a 15-page document entitled "Urogynecologic Surgical Mesh: Update on the Safety and Effectiveness of Transvaginal Placement for Pelvic Organ Prolapse" which has a literature review identifying 27 key references [2].

The FDA's aims in this update are to inform that: (1) "serious complications with surgical mesh for transvaginal repair of POP are not rare" (a change from the previous FDA notification) and (2) "it is not clear that transvaginal POP repair with mesh is more effective than traditional non-mesh repair in all patients with POP and it may expose patients to greater risk". From January 1, 2008 through December 31, 2010, using the Manufacturer and User Device Experience (MAUDE) database, the FDA received 2,874 additional reports of complications associated with surgical mesh devices used to repair POP and SUI, with 1,503 reports associated with POP repairs and 1,371 associated with SUI repairs. The scope of the current safety communication was limited to POP surgery. The FDA noted mesh exposure ("erosion") as the most common mesh-related complication and with mesh shrinkage ("contraction"), the leading cause of symptoms including bleeding, pelvic pain, dyspareunia, or apareunia.

The results of the literature review [2], summarized in the FDA Safety Communication [1], were: (1) mesh used in transvaginal POP repair introduces risks not present in traditional non-mesh surgery for POP repair; (2) mesh placed abdominally for POP repair appears to result in lower rates of mesh complications compared to transvaginal POP surgery with mesh; (3) there is no evidence that 
transvaginal repair to support the top of the vagina (apical repair) or the back wall of the vagina (posterior repair) with mesh provides any added benefit compared to traditional surgery without mesh; and (4) while transvaginal surgical repair to correct weakened tissue between the bladder and vagina (anterior repair) with mesh augmentation may provide an anatomic benefit compared to traditional POP repair without mesh, this anatomic benefit may not result in better symptomatic results.

The FDA's key concerns [1] are followed by 15 recommendations for health care providers including adequacy of training for each new procedure, patient information and counseling, risk/benefit analysis, and proper vigilance for the development of complications. There were 17 recommendations for patients before and after surgery in terms of the questions to ask their surgeon prior to any proposed pelvic floor surgery as well as the follow-up care that is optimal following such surgery.

Among clinicians in the fields of urogynecology and pelvic floor surgery, there is a wide disparity of views as to the propriety of the use of prostheses and grafts. There will be an equal variance in reactions to this new FDA safety communication. Those opposed to mesh usage in POP surgery might welcome this communication and may even suggest that the concerns might have been more strongly expressed. Those enthusiastic mesh users might regard the concerns as too strong and a potential impingement on their ability to practice using such prostheses. The very selective mesh user might see the concerns as a possible justification of their caution in "picking their cases" to use synthetic mesh products.

The academic observer will appreciate that the FDA has appeared to conscientiously evaluate the peer-reviewed scientific literature on the safety and effectiveness of surgical mesh for POP, including relevant randomized controlled trials (RCTs), systematic reviews, and selected observational studies. The eight "concerns" raised in relation to safety and six "findings" in relation to effectiveness, outlined in the abovementioned 15-page document [2], reflect that literature, though there are six limitations noted for that literature. The latter include inconsistent defining and reporting of adverse events and the paucity of studies extending beyond 2 years follow-up.

If a heightened level of debate as to the use of mesh is prompted by the FDA safety communication, greater attention to risk/benefit analysis among other factors is displayed by pelvic floor surgeons, and a greater level of information, awareness, and caution is afforded to patients, then all of these outcomes might reasonably be regarded as a positive reaction. Further positive reactions would be the stimulation of a greater level of research into (1) comparing complications from mesh-related pelvic floor procedures, by themselves, and in relation to complications from native tissue pelvic floor surgery; (2) reducing the rate of complications from mesh-related procedures; and (3) improving the efficacy of native tissue procedures.

The FDA's first "safety caution" [2] is "Patients who undergo POP repair with mesh are subject to mesh-related complications that are not experienced by patients who undergo traditional surgery without mesh". This statement can only be scientifically verified if the complications from the different surgeries can be properly classified with systems that are comparable and then the complications accurately compared. The Terminology and Standardization Committee of the International Urogynecological Association (IUGA) in collaboration with the Standardization Steering Committee of the International Continence Society (ICS) is making the first of these research aims possible. In January 2011, the IUGA-ICS joint terminology and classification of the complications related directly to the insertion of prostheses (meshes, implants, tapes) and grafts in female pelvic floor surgery was published simultaneously in this journal [3] and Neurourology and Urodynamics. Userfriendly color and laminated clinical charts were included in the publication issues of each journal. An advanced draft has been produced for review by the same authorship of the equivalent classification for native tissue female pelvic floor surgery, employing the same category $(\mathrm{C})$, time $(\mathrm{T})$, and site (S) style of classification. It is hoped this might be ready for publication early in 2012 again with color and laminated clinical charts.

In relation to the third FDA "safety concern" [2] which led to some of their key warnings [1] "Mesh-associated complications are not rare" and "the most-common meshrelated complications experienced by patients undergoing transvaginal POP repair with mesh is vaginal mesh erosion", ICS and IUGA again intend to contribute to a greater worldwide knowledge of the issue. Already an online ICSIUGA Complication Classification Calculator Code (CCCC) can be accessed from both society websites. From this, an online registry will be created seeking the input from individual members and different national urogynecological societies. The input of Mr Dominic Turner of ICS and the British Society of Urogynaecology has been particularly important. Generic (nonidentifiable to avoid medicolegal issues) data to be collated are: (1) the country of origin, (2) the nature of the operation, and (3) the CCCC. Acting as a "carrot" for individuals and national societies to contribute complication data will be the ability to access accumulated data from the registry of the overall world (assuming an increasing number of national societies link in) experience of the categories, times, or sites of the different complications. It is realized that the data gained should be multinational and hopefully in large numbers, though it will not have the scientific quality provided by a randomized trial [4].

The second positive research reaction to this and the previous FDA communication would be to try and reduce the 
rate of mesh-related complications, in particular the most common exposure-type complications seen with the use of non-absorbable mesh. The literature tends to point to average synthetic non-absorbable mesh exposure rates of $10-20 \%$ with one highly reported study at the upper end of this range [5]. Improvements in surgical technique as well as surgical materials may each need to play a part to achieve this benefit. In terms of surgical technique, full thickness vaginal wall dissection is being seen as one technique for optimal mesh placement to minimize complications. Whether a low singledigit (even $1 \%$ as suggested by a recent IUGA conference presentation [6]) synthetic mesh exposure rate is consistently possible using such techniques requires further study. It is hoped that the use of less quantity and lighter mesh might also see a reduction in exposure-related complications, though one such study recently still showed a cumulative rate of exposure of $12.5 \%$ at 2-year follow-up [7].

There is no clear differentiation in the concerns of the FDA report $[1,2]$ between synthetic non-absorbable synthetic meshes and alternate meshes such as synthetic absorbable and biological (absorbable) meshes. There is some evidence that the use of biologic meshes by those pelvic floor surgeons who will not use non-absorbable meshes may be an advantage in terms of anatomical success over native tissue surgery with fewer complications than nonabsorbable meshes [8]. However, good evidence on subjective efficacy is lacking.

The third positive research reaction to FDA warnings is to improve the results from native tissue surgery to such a degree that the use of prostheses and grafts is far less indicated. The FDA's literature review on effectiveness of POP with mesh largely concurs with equivalent Cochrane data [9]. Noted in two FDA "effectiveness" points [2] is the failure of the mesh to provide significant anatomic benefit in the posterior compartment over the equivalent native tissue surgery. Noted in one FDA "effectiveness" point (and Cochrane data) is the anatomical though not necessarily symptomatic benefit from mesh augmentation in the anterior compartment. Concentration of anterior compartment research should be aimed at improving the results of native tissue anterior colporrhaphies. As it has been shown that around half of anterior vaginal wall descent can be explained by the degree of vaginal vault descent present [10], research into newer native tissue techniques for anterior compartment POP repairs may need to involve concomitant anterior vaginal vault support.

Reactions to the FDA recent statements can be positive in the medium term. Hopefully in time, the greater level of publicity and debate on the issue of mesh augmentation of prolapse surgeries might lead to more research (1) to reducing mesh-related complications and (2) to improving the results of native tissue surgeries. This would lead to a greater level of vindication for all sides of the debate. The IUGA and ICS Terminology Classification of Complication Reports for the different types of pelvic floor surgeries, an upcoming IUGA-ICS report on outcomes of surgery for female POP, as well as the ICS-IUGA online complication registry are all designed to assist the production and monitoring of such research. This research should ideally include large RCTs such as the United Kingdom's PROSPECT trial (https://viis.abdn.ac.uk/HSRU/prospecta large trial looking at the symptomatic and objective results of the use of (1) native tissues alone, (2) additional biological implants, and (3) additional synthetic nonabsorbable materials in primary and secondary POP surgeries). This might then also result in the closure of the clinical governance gap between the data required for the introduction of synthetic products for female POP surgery and that required for the introduction of new drugs. Instead of an upgrade of the FDA safety communication from 2008 to 2011 , let us also hope the combination of the positive reactions outlined might even see the possibility of a future FDA downgrade of any current concerns on this subject.

\section{References}

1. FDA (2011) FDA Safety Communications: UPDATE on serious complications associated with transvaginal placement of surgical mesh for pelvic organ prolapse. http://www.fda.gov/MedicalDevices/ Safety/AlertandNotices/ucm262435.htm. Accessed 13 July 2011

2. Urogynecologic surgical mesh: Update on the safety and effectiveness of transvaginal placement for pelvic organ prolapse (2011) http://www.fda.gov/MedicalDevices/Safety/AlertandNotices/ ucm262760.pdf

3. Haylen BT, Freeman RM, Swift SE, Cosson M, Davila GW, Deprest J, Dwyer PL, Fatton B, Kocjancic E, Lee J, Maher C, Petri E, Rizk DE, Sand PK, Schaer GN, Webb RJ (2011) An International Urogynecological Association (IUGA)/International Continence Society (ICS) joint terminology and classification of the complications related directly to the insertion of prostheses and grafts in female pelvic floor surgery. Int Urogynecol J 22:3-15

4. Roovers J-PWR (2007) Registries: what level of evidence do they provide? Int Urogynecol J 18:1119-1120

5. Inglesia C, Sokol AI, Sokol ER, Kudish BI, Gutman RE, Peterson JL, Shott S (2010) Vaginal mesh for prolapse: a randomized controlled trial. Obstet Gynecol 116(2):293-303

6. Cassidenti AP (2011) A reproducible technique to perform full thickness vaginal wall dissection for POP mesh placement that minimizes mesh exposure rates. IUGA Lisbon abstracts. http:// www.abstractsonline.com/plan/AuthorIndex.aspx?letters=CA. Accessed June 2011

7. Milani AL, Lucente V, Urquhart C, Hinoul P, Cosson M (2011) Two-year clinical outcomes of a trocar-guided transvaginal mesh repair utilizing a new light-weight synthetic mesh. Int Urogynecol J 22(Suppl 1):S26

8. Jia X, Glazener C, Mowat G, MacLennan G, Bain C, Fraser C, Burt J (2008) Efficacy and safety of using mesh or grafts in surgery for anterior and/or posterior vaginal wall prolapse: systematic review and meta-analysis. Br J Obstet Gynaecol 115(11):1350-1361

9. Maher C et al (2010) Surgical management of pelvic organ prolapse in women. Cochrane Database Syst Rev 4:CD004014

10. Summers A, Winkel LA, Hussain HK, DeLancey JOL (2006) The relationship between anterior and apical compartment support. Am J Obstet Gynecol 194:1438-1443 